In der Rubrik „Literatur kompakt" dieser Ausgabe referieren und kommentieren diese Experten für Sie Arbeiten aus der internationalen Fachliteratur.

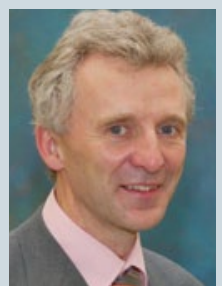

Dr. med. Martin Claßen, Bremen

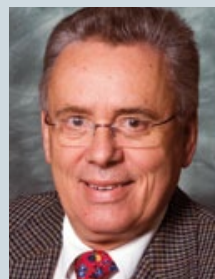

Dr. med Hartmut Koch, Vechta

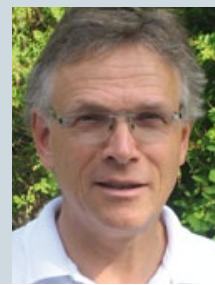

Dr. med. Ulrich Mutschler, Hildesheim

\section{Probate Probiotika: ein Mittel bei Säuglingskoliken}

\section{Säuglingskoliken gehören zu den schwierigen Themen in der Pädiatrie. Eine Gruppe von Pädiatern aus Polen testete nun Lactobacillus reuteri DSM 17938 im Vergleich zu Placebo bei betroffenen Babys, die gestillt wurden. Die Probiotika konnten die Wissenschaftler überzeugen.}

$\mathrm{D}^{2}$ e Palette der Erklärungen für Säuglingskoliken ist breit - von kopfgelenkinduzierten Symmetriestörungen bis zu einer gestörten Eltern-Kind-Interaktion. Ebenso breit ist auch das Spektrum an Therapien: Unter anderem werden immer wieder Interventionen über die Ernährung propagiert. In einer aktuellen Studie wurden 80 Kinder unter fünf Monaten, die die Kriterien einer Säuglingskolik erfüllten und gleichzeitig ausschließlich oder vorwiegend gestillt wurden, randomisiert. Sie wurden entweder mit $10^{8}$ kolonieformenden Einheiten Lactobacillus reuteri DMS 17938 oder Placebo behandelt. Die Therapie wurde einmal täglich für 21 Tage appliziert. Nachuntersucht wurde die Zeitdauer des Schreiens in Minuten pro Tag nach 7, 14, 21 und 28 Tagen.

Die Häufigkeit des Ansprechens auf die Therapie war in der Probiotika-behandelten Gruppe signifikant höher gegenüber dem Placebo und dies zu allen untersuchten Zeitpunkten. Darüber hinaus war die mittlere Dauer des Schreiens signifikant niedriger in der mit Probiotika behandelten Gruppe: Von einer Baseline von 240 Minuten pro Tag in beiden Gruppen reduzierte sich die Schreidauer bei diesen Kindern an Tag 14 auf 105 Minuten (versus 150 bei den Kontrollen) und auf 52 Minuten an Tag
28 (versus 120 Minuten). Eine Limitierung der Studie war, dass die Schreidauer nur anhand eines Tagebuches der Eltern erfasst wurde und nicht mit Rekordern.

Szajewska $\mathrm{H}$ et al. Lactobacillus reuteri DSM 17938 for the management of infantile colic in breastfed infants: a randomized, double-blind, placebo-controlled trial. J Pediatr 2013; 162 : 257-62

Kommentar: Nach einer vorangegangenen Vergleichsstudie zwischen Lactobacillus reuteri und Dimeticon, deren methodische Qualität etwas schlechter war [Savino F et al. Pediatrics 2007; 119: e124-30], zeigt auch die vorliegende Studie einen deutlichen Effekt der Applikation von Lactobacillus reuteri bei gestillten Kindern. Die Studie unterstützt die Meinung, dass Säuglingskoliken wirklich etwas mit dem Darm zu tun haben, also der Pädiater oder pädiatrische Gastroenterologe der richtige Ansprechpartner der Eltern ist. Wie zu erwarten, reagiert auch die Placebogruppe mit einer deutlichen Besserung, aber die Unterschiede sind trotzdem groß. Diese Evidenz spricht trotz der Harmlosigkeit der Störung für eine Behandlung - zumindest bei den Kindern, bei denen ein großer Leidensdruck der Eltern besteht. Der Rat zur Anwendung von Lactobacillus reuteri enthebt aber den Pädiater nicht von der Notwendigkeit, auf-

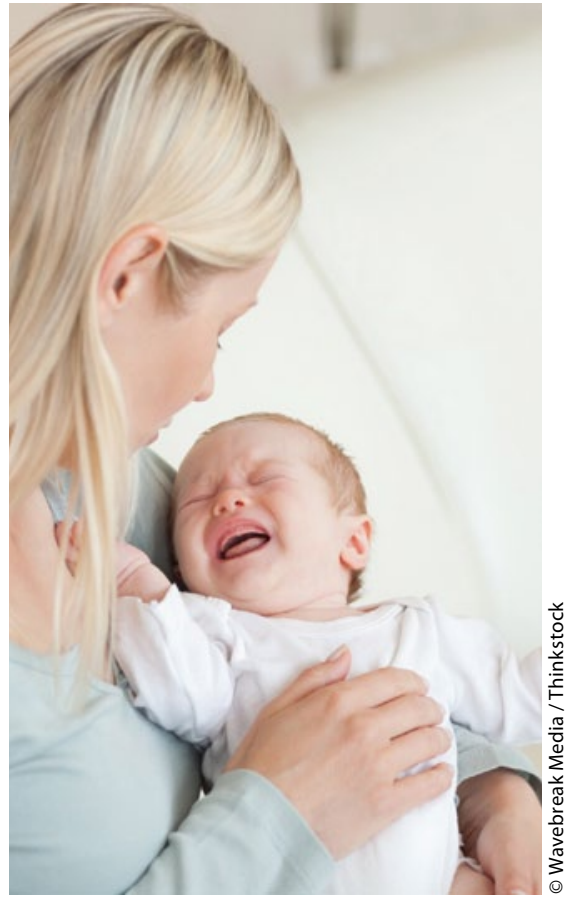

Ist der Leidensdruck der Eltern groß, könnten Probiotika zum Einsatz kommen.

merksam nach Störungen der Eltern-KindInteraktion zu schauen.

Erfreulicherweise ist damit diese Therapie besser belegt als Osteopathie und chiropraktische Therapien: Eine jüngst publizierte Cochrane-Analyse, die diese beiden Therapieoptionen ins Visier nahm, kommt bei der Analyse von sechs Studien zu dem Schluss, dass bei einer strengen Auswahl von Untersuchungen, bei denen auch die Eltern "geblindet" worden waren, kein signifikanter Effekt zu vermelden war. Auch bei diesen Maßnahmen war der Placeboeffekt bei den Eltern hoch [Dobson et al. Cochrane Database Syst Rev 2012; 12: CD004796].

Dr. Martin Claßen 